\title{
Knowledge of Hearing Loss among University Students Pursuing Careers in Health Care
}

DOI: $10.3766 /$ jaaa.17071

\author{
Erika S. Squires* ${ }^{*}$ \\ Lori A. Pakulski $\dagger$ \\ Jennifer Glassman $\dagger$ \\ Emily Diehm $\dagger$
}

\begin{abstract}
Background: Albeit limited, research suggests that students pursuing careers in health care receive limited training on the provision of services for people with hearing loss. As the incidence of hearing loss continues to increase among Americans, it is critical that medical professionals understand how hearing loss among patients may affect the manner in which they can provide services most effectively.

Purpose: The aim of this project is to assess the amount of experience and confidence that preprofessional health-care students at one university obtain during the course of their training and whether these students would be interested in additional information related to hearing health.

Research Design: Preprofessional health-care students in terminal degree programs at one university completed a survey regarding the provision of services for individuals with hearing loss. Students were asked to quantify their prior training on topics related to hearing loss, report their perceptions of the benefits and barriers to screening hearing, and report their self-efficacy in providing services for individuals with hearing loss. Additional survey items investigated students' interest in receiving further training on these topics.

Study Sample: Participants ( $n=95 ; 16.2 \%$ response rate) were students at a mid-sized, Midwestern university who were pursuing the following terminal degrees: medicine, physician assistant, nursing, pharmacy, physical therapy, occupational therapy, speech-language pathology, and respiratory therapy (bachelor's degree in respiratory care). Participants were selected based on membership in an interdisciplinary education training program. All students in this program received an invitation to participate in the study. Of the participants, 68 (71.6\%) were Caucasian and 86 (90.5\%) were female.

Data Collection and Analysis: A 28-item online survey on various topics related to hearing loss was used to document student responses. All students enrolled in a university's interdisciplinary professional education course $(n=586)$ received an online link to the survey via an initial email, which contained a brief introduction to the study, the assurance of response anonymity, and a statement regarding implied consent. A second email was sent to students, which reminded participants of the request to complete the survey.

Results: Overall, $60 \%$ of participants reported an interest in receiving additional information on hearing health and $66.3 \%$ of respondents indicated that they wished to receive training via an in-service or internet workshop facilitated by their university program. Most of the participants reported that they had not received training and did not feel confident identifying the signs and symptoms of hearing loss and making an appropriate referral, which led to the request for additional information.
\end{abstract}

*Department of Communication Sciences and Disorders, Wayne State University, Detroit, MI; $†$ Speech Language Pathology Program, University of Toledo, Toledo, $\mathrm{OH}$

Corresponding author: Lori A. Pakulski, Speech-Language Pathology Program, Department of Rehabilitation Sciences, College of Health Sciences, The University of Toledo, Toledo, OH 43606; Email: lori.pakulski@utoledo.edu

Portions of this paper were presented as a poster at the Michigan Speech Language Hearing Association Annual Convention, Grand Rapids, MI, April 21-23, 2016.

The contents of this research were developed under a grant from the Department of Education. However, these contents do not necessarily represent the policy of the Department of Education and you should not assume endorsement by the Federal Government.

Ethical approval has been waived by the University of Toledo Institutional Review Board: Protocol \# 0000201092; Approval Date: 2/12/16. 


\begin{abstract}
Conclusions: The results of this study suggest that preprofessional health-care students have an interest in receiving additional education on various topics related to hearing loss including a better understanding of how hearing loss impacts the quality of life in affected individuals. These findings provide an incentive to provide additional training related to hearing loss identification and management for preprofessional health-care students to foster increased competency and improved patient care.
\end{abstract}

Key Words: education, health care, hearing loss, student

Abbreviations: IPE = interprofessional education; NIHL = noise-induced hearing loss; SLP = speechlanguage pathology; SLPs = speech-language pathologists

\section{INTRODUCTION}

$\mathrm{H}$ earing loss is a public health issue with as many as $20 \%$ of Americans reporting some degree of hearing loss (HLAA, 2015). The NASEM (2016) reports that as many as $67-86 \%$ of individuals in the United States (ages $\geq 50$ ) who could benefit from hearing technology do not make use of these devices. On a global scale, the World Health Organization has deemed hearing loss to be a burden with substantial social and economic ramifications (Duthey, 2013). What is particularly troubling is that the incidence of hearing loss (Schoenborn and Heyman, 2008; Shargorodsky et al, 2010) and the frequency of health, psychosocial, and learning problems (e.g., attention-deficit/hyperactivity disorder and expressive and receptive language concerns) associated with hearing loss (Kochkin and Rogin, 2000; Pittman et al, 2009; Daud et al, 2010; Hogan et al, 2011; Lin et al, 2011) are increasing among Americans. Despite mounting concerns, relatively few school-aged children and adults are screened or evaluated routinely for hearing loss (Davis et al, 2007; Hendershot et al, 2011 ). For example, $<12 \%$ of physicians screen hearing (Kochkin, 2005) or refer for hearing evaluations routinely (Cohen et al, 2005). School nurses report that most school-age students are not screened past ninth grade (Hendershot et al, 2011), which is becoming increasingly important as the incidence of noise-induced hearing loss has increased in prevalence among those ranging in age between 6 and 19 years old from 14.9\% to 19.5\% (Shargorodsky et al, 2010).

Although audiologists and speech-language pathologists routinely screen and/or evaluate patients' hearing, many Americans do not seek services from these professionals. Consequently, other health-care professionals (e.g., occupational and physical therapists, nurses, physician assistants, and physicians) must possess the knowledge, skills, and experience necessary to collaborate with one another to provide each individual with the appropriate referral for diagnosis, treatment, and management for his or her hearing loss.

The concern is not only in identifying hearing loss, but also obtaining the appropriate referral source for intervention. On average, people wait 7 years from the time they suspect hearing loss before seeking help from a medical professional (HLAA, 2015). Furthermore, according to the NIH (2010), 80\% of adults who could benefit from hearing technology do not seek intervention. This is problematic because people with unmanaged hearing loss are at greater risk for diminished psychological and physical health, impaired memory, reduced academic or vocational performance, and withdrawal from social situations (Kochkin, 2013). Conversely, a study conducted by the Department of Veterans Affairs, National Institute on Deafness and Other Communication Disorders and contributing authors, and published in the Journal of the American Medical Association, provided evidence of the benefits of amplification in ameliorating many of these negative effects (Larson et al, 2000). In addition, a recent study conducted by researchers at Indiana University, and published in the American Journal of Audiology, provided evidence that when older adults receive hearing aids from qualified and knowledgeable providers who use audiology-based best practices, this technology improved users reports of "hearing aid benefit, satisfaction, and usage" (Humes et al, 2017). Thus, it is critical to find ways to increase the number of children and adults who obtain routine hearing screenings, and follow-up with early management, when needed.

To meet the needs of patients with hearing loss, those suspected to have hearing loss, and those at risk for noise-induced and other kinds of preventable hearing loss, health-care professionals must have the following:

- the ability to recognize the signs and symptoms of hearing loss

- the ability to effectively communicate with patients who have untreated or poorly managed hearing loss in their own course of practice

- an understanding of the consequences of living with hearing loss and the impact that it can have on a person's quality of life if left untreated

- the ability and resources necessary to provide an appropriate referral for a person with suspected hearing loss

- an understanding of how to work as part of a collaborative team to provide best-practice care for individuals with hearing loss.

Another important aspect of a health-care provider's role in hearing health is preventative education. It is important that health-care professionals have the knowledge 
and resources needed to provide their patients with a thorough understanding of how to reduce the risk of acquiring noise-induced hearing loss (NIHL). It is estimated that approximately $15 \%$ of the population between the ages of 20 and 69 have hearing loss that may have been caused by vocational or recreational exposure to noise (NIDOCD, 2015). Common recreational causes of NIHL include shooting guns, riding loud vehicles, listening to MP3 players loudly through earbuds or headphones, attending loud concerts, and mowing the lawn without hearing protection, as examples. Health-care providers should have an understanding of the types of environments and behaviors that increase someone's likeliness of acquiring NIHL, so they are able to counsel their patients on the importance of avoiding these situations or using proper hearing protection when noise exposure cannot be avoided (e.g., in the workplace).

\section{PURPOSE}

A lbeit limited, research has suggested that students who are preparing for careers in health care are underprepared to assist and refer patients with hearing loss to the proper professional. Yet, it is hypothesized that there is a potential benefit from additional training related to hearing health.

Physicians and other health-care providers are in a position to address hearing concerns with the patients. Yet, little is known about their education concerning the provision of services for people with hearing loss. The aim of this study was to describe preservice health-care providers' knowledge, competence, and confidence regarding best-practice services for patients with hearing loss.

\section{RESEARCH DESIGN}

\section{Study Sample}

Preprofessional health-care students enrolled in an Interprofessional Education (IPE) program at a Midwestern university served as participants. A total of
586 students pursuing the following degrees: medicine, physician assistant, nursing, pharmacy, physical therapy, occupational therapy, speech-language pathology, and respiratory therapy (those pursuing a bachelor's degree in respiratory care) received the request to participate. Of the 586 students who were invited to participate in the study via email, 95 opted to participate for a response rate of $16.2 \%$. A majority of the responding students reported that they were Caucasian (71.6\%) and female $(90.5 \%)$. Other demographic and background characteristics of respondents can be seen in Table 1.

\section{DATA COLLECTION AND ANALYSIS}

\section{Instrument}

A 28-item online survey regarding hearing loss, screening, and prevention was adapted from a previously published instrument that explored school nurses' knowledge and practice related to hearing loss (Hendershot et al, 2011). The survey was designed to assess the current status of knowledge and skill among preprofessional health-care students. The instrument consisted of background characteristics, including program, level of education, age, gender, ethnicity, and personal hearing status. Survey items dealt with questions on perceptions and practices regarding screenings, perceptions of interventions and education, and the amount of training that students have received on various topics related to hearing health. Content validity of the survey instrument along with test-retest reliability was addressed in the Hendershot et al (2011) study.

The survey included items that used Stages of Change theory (precontemplation, contemplation, preparation, action, and maintenance) (Prochaska et al, 1992) to assess participants' readiness to provide patients with followup for potential hearing loss. In addition, two key constructs of the Health Belief Model were operationalized: perceived barriers $(n=5$ items) and perceived benefits ( $\mathrm{n}=5$ items) for providing hearing loss prevention

Table 1. Participant Demographics and Response Rates

\begin{tabular}{lcccc}
\hline Program & $N$ & \% of Total Sample $(n=95)$ & Response Rate of Program (\%) & Year in School \\
\hline Speech-language Pathology & 40 & 42.10 & 81.10 & G: Year 1 \\
Other Programs & & & & \\
Medical School & 11 & 11.58 & 6.20 & G: Year 1 \\
Physician Assistant & 3 & 3.16 & 7.80 & G: Year 1 \\
Nursing & 6 & 6.32 & 2.80 & UG: Year 1 \\
Pharmacy & 3 & 3.16 & 17.90 & G: Year 1 \\
Physical Therapy & 5 & 5.26 & 30.00 & G: Year 1 \\
Occupational Therapy & 6 & 6.32 & 5.60 & G: Year 1 \\
Respiratory Therapy & 1 & 1.05 & & UG: Year 1 \\
No Program Listed & 20 & 21.05 & 16.20 & \\
Total & 95 & 100.00 & &
\end{tabular}

Note: UG = undergraduate student; $\mathrm{G}=$ graduate student. 
training (Janz and Becker, 1984). Perceived benefits and barriers were used in the survey because these constructs have been found to be the most significant predictors of behaviors within the Health Belief Model (Harrison et al, 1992). Also included were perceptions ( $\mathrm{n}=7$ items) of hearing loss prevention activities such as accuracy in screening and tracking hearing loss over time, providing appropriate referrals, and background/ demographic questions. Most questions on the survey were closed-format questions with multiple response categories. Likert-type scales (e.g., not confident to very confident) were used to assess preprofessional health-care students' perceptions and beliefs on various topics related to hearing health.

\section{Procedures}

This study was reviewed by the social, behavioral, and educational Institutional Review Board (IRB) and deemed to be exempt from our IRB because of the similarity of the instrument's content to other academic work the students already perform within their programs. Participants received an online link to the survey via an initial email, which contained a brief introduction to the study, the assurance of response anonymity, and a statement regarding implied consent. A second email was sent to students, which reminded participants of the request to complete the survey. Students were not offered any financial incentive to complete the survey.

\section{Data Analysis}

Descriptive statistics with a report of the frequencies, means, and standard deviations were used to describe the responses to the questionnaire items, as well as the demographic and background characteristics of the respondents.

\section{RESULTS}

\section{Number of Hours of Coursework Related to Hearing Loss}

Students were queried concerning the number of hours of coursework they have received on hearing health (Figure 1). The majority of SLP students (81.6\%) reported that they have received five or more hours of coursework on various topics related to hearing loss identification, prevention, and management; however, no participating students $(0 \%)$ in other health-care programs reported receiving five or more hours of coursework on these topics. In fact, $45.7 \%$ of students in other health-care programs indicated that they have not received any coursework on hearing loss identification, prevention, and management.

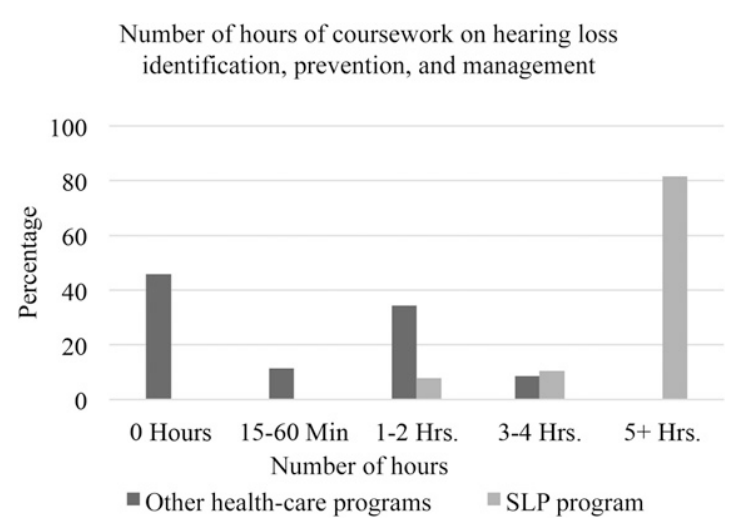

Figure 1. Number of hours of coursework on hearing loss identification, prevention, and management.

\section{Hearing Screening Efficacy Expectations}

Students were asked to report on their self-efficacy regarding identifying potential hearing concerns and making a referral for a patient with suspected hearing loss (Figures 2 and 3). It is important to note that the survey did not ask students to report on their selfefficacy in using specific tools for hearing loss screening (e.g., instrumental versus checklist), but instead kept the definition of "hearing loss screening" open to students for interpretation.

The majority of responding preprofessional SLP students $(92.1 \%)$ indicated that they were "confident" or "very confident" in their ability to accurately screening a patient's hearing, whereas approximately half of students $(51.4 \%)$ in preprofessional health-care programs other than the SLP program indicated that they were "not confident" in their ability to screen a person's hearing.

Students were also asked to report on their selfefficacy regarding providing an appropriate referral for a patient at risk for hearing concerns (Figure 3). Approximately three-fourths of SLP students (78.9\%) indicated that they were "confident" or "very confident" in their ability to provide an appropriate referral for a patient

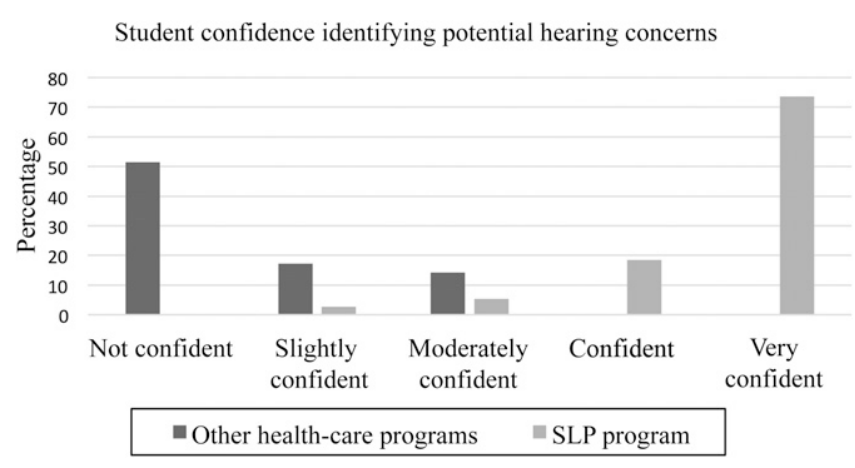

Figure 2. Student-reported self-efficacy for identifying potential hearing concerns. 
Student confidence making referrals for patients with suspected hearing loss

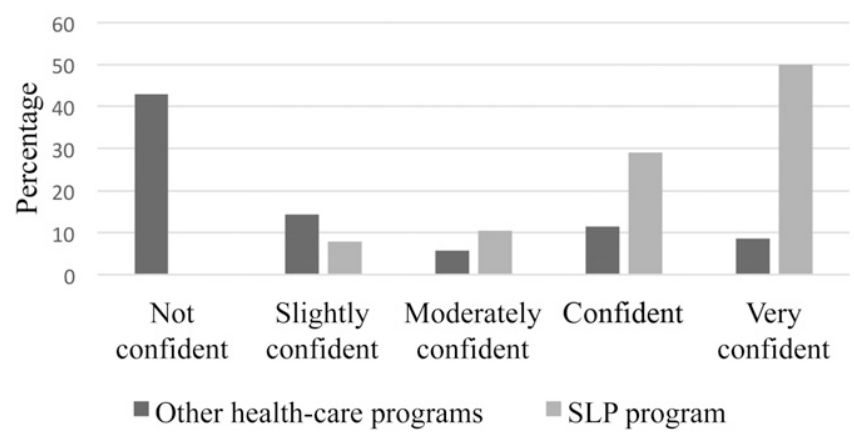

Figure 3. Student-reported self-efficacy for providing referrals for patients with suspected hearing loss.

with hearing concerns. Conversely, $42.9 \%$ of students in other health-care programs reported that they were "not confident" in their ability to provide an appropriate referral for a patient with suspected hearing loss.

\section{Perceived Benefits of Screening for Hearing Loss}

Students also reported on their perceived benefits of screening individuals for hearing loss (Table 2). The top three perceived benefits of screening hearing among SLP students were (a) screening provides information to the patient, parent, or caregivers, so they can contribute to prevention efforts (95\%), (b) screening creates awareness of hearing loss problems (95\%), and (c) screening provides support for programs to address preventable hearing loss $(90 \%)$. The top three perceived benefits of conducting hearing screenings among students enrolled in other programs were (a) screening increases the quality of life for individuals (89.1\%), (b) screenings provide information to the individual, parents, and caregivers, so they can contribute to prevention efforts (85.5\%), and (c) screening provides support for programs to address preventable hearing loss $(80 \%)$.

\section{Perceived Barriers to Screening Patients' Hearing}

Participants responded to items regarding their perception of potential barriers that may prevent healthcare providers from screening patients' hearing (Table 2). Among SLP students, the top three perceived barriers to screening hearing were (a) not having the proper equipment or knowing what tools to use to screen hearing

Table 2. Summary of Students' Perceptions of Benefits and Barriers to Screening Patients for Hearing Loss and Barriers to Providing Appropriate Follow-Up

\begin{tabular}{|c|c|c|}
\hline & \%SLP Students & $\%$ Students in Other Programs \\
\hline & $n=40$ & $n=55$ \\
\hline \multicolumn{3}{|l|}{ Benefits of screening patients for hearing loss } \\
\hline There are no benefits to screening patients for hearing loss & 0 & 0 \\
\hline Increases quality of life for individuals & 80 & 89.1 \\
\hline $\begin{array}{l}\text { Provides information to the individual, parents, or caregivers so they can } \\
\text { contribute to prevention efforts }\end{array}$ & 95 & 85.5 \\
\hline Creates awareness of hearing problems & 95 & 60 \\
\hline Provides support for programs to address preventable hearing loss & 90 & 80 \\
\hline \multicolumn{3}{|l|}{ Barriers that may prevent health-care providers from screening patients' hearing } \\
\hline There are no barrier to screening patients for hearing loss & 32.50 & 9.1 \\
\hline There is a lack of interest/response & 20 & 38.1 \\
\hline Do not have enough time & 10 & 25.5 \\
\hline Do not know how to properly screen for hearing loss & 7.50 & 65.5 \\
\hline Do not know how to properly screen for noise-related hearing loss & 15 & 54.5 \\
\hline $\begin{array}{l}\text { Do not have adequate resources and/or referral sources for patients with } \\
\text { hearing problems }\end{array}$ & 27.50 & 50.9 \\
\hline Do not have proper equipment or know what tools to use to screen hearing & 40 & 56.4 \\
\hline \multicolumn{3}{|l|}{ Barriers to providing appropriate follow-up for hearing concerns } \\
\hline $\begin{array}{l}\text { Lack of time to focus on patients who may be at risk because of other job } \\
\text { responsibilities }\end{array}$ & 35 & 52.7 \\
\hline Lack of expertise on noise-related hearing loss prevention & 52.50 & 74.5 \\
\hline Lack of standardized material for patients and/or caregivers & 45 & 47.3 \\
\hline Lack of resources available to patients who may be at risk & 47.50 & 47.3 \\
\hline $\begin{array}{l}\text { The issues of noise-induced hearing loss and prevention are minor compared } \\
\text { with other issues addressed by health-care providers }\end{array}$ & 35 & 41.8 \\
\hline $\begin{array}{l}\text { Inability to affect patients' behavior related to personal listening devices or other } \\
\text { cause of noise-induced hearing loss }\end{array}$ & 75 & 50.9 \\
\hline
\end{tabular}

Note: Percentage on chart represents percentage of students who agreed with the statement. 
(40\%), (b) not having adequate resources and/or referral sources for patients with hearing problems (27.5\%), and (c) a lack of patient interest/response (20\%).

The top three barriers that students in other health-care programs reported may prevent a health-care provider from screening patients' hearing were (a) not knowing how to properly screen for hearing loss $(65.5 \%)$, (b) not having the proper equipment or knowing which tools to use to screen hearing (56.4\%), and (c) not knowing how to properly screen for noise-related hearing loss in particular $(54.5 \%)$.

In addition, $32.5 \%$ of SLP students responded that they felt there were no barriers that might prevent a health-care provider from screening patients' hearing. Of students enrolled in other health-care programs, $9.1 \%$ of students responded that they felt there were no barriers to screening.

\section{Perceived Barriers to Providing Appropriate Follow-Up for Hearing Concerns}

Students were asked to identify potential barriers that may prevent health-care providers from providing appropriate follow-up care for hearing concerns (Table 2). Responding SLP students reported an inability to affect a patient's behavior related to personal listening devices and/or other causes of noise-induced hearing loss $(75 \%)$, a lack of expertise on noise-related hearing loss prevention $(52.5 \%)$, and a lack of resources available to patients who may be at risk for hearing loss $(47.5 \%)$ as the top three perceived barriers.

Students in other health-care programs responded that a lack of expertise on noise-related hearing loss prevention (74.5\%), a lack of time to focus on patients who may be at risk for hearing loss because of other job responsibilities $(52.7 \%)$, and an inability to affect a patient's behavior related to personal listening devices and/or other causes of noise-induced hearing loss (50.9\%) as the top three barriers that may prevent health-care providers from providing the appropriate follow-up care for hearing concerns.

\section{Students' Interest in Receiving Additional Information on Hearing Loss}

Students indicated whether they were interested in receiving additional information on hearing loss identification, management, and prevention. Approximately two-thirds of responding students in health-care programs other than the SLP program $(65.7 \%)$ indicated that they were interested in receiving additional information and $57.9 \%$ of SLP students reported an interest in further training on these topics. When queried about the manner in which they wished to receive additional information related to identify, managing, and preventing hearing loss, the students reported the greatest interest in receiving additional training via in-service workshops $(66.67 \%)$ and the internet $(66.67 \%)$.

\section{Limitations}

Findings from this project should be interpreted with consideration of the potential limitations of this study. First, the data were self-reported, and some of the student participants may have over- or underreported some of their responses. If this occurred, the results may make it appear as if students in preprofessional health-care programs at this university receive more or less training than they actually do. Participants may have wanted their future profession to be represented in a positive manner and may have given more socially desirable responses. If this occurred, this would be a threat to the internal validity of the findings.

The response rate could also be a threat to the generalizability (i.e., external validity) of the findings considering that those who did not participate in the survey may have responded differently from those who did participate. This survey was led by an SLP student, so students in this program were more likely to respond. Forty-two percent of the respondents were SLP graduate students and this high response rate from one particular healthcare program could also limit the external validity of the findings. However, it is worth noting that responses from SLP students were analyzed separately from the responses provided by students in other health-care programs. Of additional importance is the consideration that analyzing results based on a student's length of participation in his/her respective program may have added strength to the statistical analysis and interpretation of data, as it is possible that coursework on these topics is introduced later into a student's training.

The survey used was adapted from a previously published instrument that explored school nurses' knowledge and practice related to hearing loss (Hendershot et al, 2011). Actual focus groups with health-care providers were not used to develop and select items for the survey. It is possible that such focus groups could have revealed other equally important issues not included in the questionnaire. If so, this too could threaten the applicability of the results.

\section{DISCUSSION}

I n summary, the majority of preprofessional healthcare students (in programs other than the SLP program) reported that they have not received coursework or experience related to hearing loss prevention and management. Most students who participated in this study expressed an interest in obtaining additional training on these topics.

It is critical that health-care professionals have the ability to identify potential hearing concerns and make 
appropriate referrals when hearing loss is suspected; otherwise, these professionals may be providing information and instructions to patients who cannot adequately hear them or who may not have the cognitive power to understand them, or a way to determine the difference. This has the potential to significantly impact the quality of services provided by medical professionals. Whereas most of the SLP students reported that they were confident in their ability to identify potential hearing concerns and make appropriate referrals for individuals with suspected hearing loss, most of the students in other health-care programs did not. It is critical that medical professionals have the skills and training necessary to identify potential hearing concerns that may affect communication and their patients' medical care, as well as the ability to make appropriate referrals for patients at risk for hearing loss. Evidence has suggested that health-care providers may not receive adequate coursework during their educational training to meet the complete needs of patients with hearing loss. Audiologists are in a position to provide education and training to other professionals both at the university level and for coworkers, and in some cases, to provide tools (i.e., hearing loss questionnaires) so that a wider range of health professionals are prepared to screen and refer.

Students indicated they may not be able to complete hearing screenings because of other job responsibilities. This student-perceived barrier is supported by practicing physicians who report that they do not routinely complete hearing screenings for patients (Cohen et al, 2005; Kochkin, 2005). This suggests that some health-care providers do not perceive hearing screening as a priority. However, research has revealed that a person generally waits 7 years from the time they suspect hearing loss before seeking help from a medical professional (Shargorodsky et al, 2010). Therefore, health-care providers should not assume that if patients do not report concerns about potential hearing loss, they are not experiencing the associated symptoms. Again, audiologists are positioned to educate other health-care professionals about this research, and provide training in ways to address screening and referral, and particularly how best to work with a patient who is suspected of untreated hearing loss until it can be addressed.

In regard to the anticipated barriers to appropriate follow-up reported by participants, all of these potential obstacles can be eliminated if a provider knows how and when to refer to an audiologist who has received specialized training to address these issues. This finding also highlights the important role of audiologists to educate health-care providers on indications for referral.

Related to this, medical professionals must carefully consider the issues of effectively communicating with patients who have untreated or poorly managed hear- ing loss about their health care. Consider the following questions:

- How does a physical therapist provide appropriate instructions in a noisy gym setting?

- How does a physician ensure that a patient clearly understands the risks and benefits of a procedure if it is explained to a patient in a hospital room with loud, beeping machines?

- How does an SLP comment on the adequacy of a person's auditory comprehension if he or she has not screened the patient's hearing first?

Audiologists could ask these types of questions of each health-care provider to insure their ability to provide patients with the highest quality care if their hearing has not been screened. Although unmanaged hearing loss is associated with diminished psychological and physical health among several other risk factors, the quality of health care received by patients with unmanaged hearing may also be reduced. Related to this topic, research has indicated that there is a relationship between hearing loss and family member concerns of safety, including a person's ability to hear instructions from physicians (NIH, 2010).

\section{Implications for Future Training}

The results of this study suggest that preservice health-care students could benefit from additional training and education on hearing loss and how it affects quality of life in affected individuals. They also would benefit from learning to effectively communicate with patients who have untreated or poorly managed hearing loss. To address these issues, students in university settings and health-care providers should be provided training and tools, and audiologists are best positioned to provide training and advocate for these changes. For example, those students who are pursuing careers in health care at this university will be offered an online in-service related to hearing loss identification and management through the university's IPE program. Audiologists can promote this type of educational opportunity at other universities as well as in their workplace.

\section{CONCLUSIONS}

A $\mathrm{s}$ the US population rapidly ages, health professionals must be equipped with the skills needed to identify and manage hearing loss, as well as to provide preventative education. In addition, the incidence of preventable hearing loss caused by noise exposure is increasing among children because of increased use of personal audio devices, including smartphones (Hendershot et al, 2011; HLAA, 2015). 
Hearing loss is a public health epidemic that has potentially devastating consequences on the physical and mental health of those affected. Medical professionals should screen their patients on a regular basis for hearing loss, and they should have the skills and training necessary to make an appropriate referral and advocate for people suspected to have hearing loss. It is imperative that health-care professionals receive training on topics related to hearing health during their educational preparation, and audiologists are best positioned to advocate for these changes and provide the necessary training. However, those who do not receive this training would benefit from seeking out a way to obtain these important skills. This project aimed to describe the preservice health-care professionals' knowledge, competence, and confidence regarding providing best-practice care for patients with hearing loss with long-term goal of creating resources to help medical professionals develop these skills during the course of their education. This project was also undertaken in an effort to educate audiologists about the need for them to advocate and educate their current and future coworkers.

There is a critical need for routine hearing screenings and follow-up treatment for individuals at risk for developing and affected by hearing loss because research suggests that people with poorly managed hearing loss are at risk for decreased physical health, mental health, and general quality of life (Kochkin, 2013). Health-care providers in all fields share a responsibility to optimize the services they provide to maximize patients' general health and well-being, both of which are compromised by unidentified or poorly managed hearing loss. Health-care providers cannot adequately address the health concerns of patients who cannot hear them.

Although identifying and contributing to the management of hearing loss may not specifically appear in health professionals' scopes of practice, allusions to this responsibility may exist. For example, the American Occupational Therapy Association (n.d.) states that an occupational therapist "addresses the physical, psychological, and cognitive aspects of their well-being through engagement in occupation." Individuals should be able to look for signs of hearing loss and realize the impact that it could have on an individual's ability to communicate with health-care providers.

The goal of this project is to provide a stepping stone from which additional training related to hearing loss identification and management can be provided to preprofessional health-care students to foster increased competency and improved patient care.

Acknowledgments. The authors wish to thank the IPE team who sent the emails to students and to the participants who decided to take the time to respond to this survey.

\section{REFERENCES}

American Occupational Therapy Association. (n.d.) Occupational Therapy: Improving Function While Controlling Costs. http:// www.aota.org/About-Occupational-Therapy/Professionals.aspx. Accessed July 25, 2016.

Cohen SM, Labadie RF, Haynes DS. (2005) Primary care approach to hearing loss: the hidden disability. Ear Nose Throat J 84(1): $26-31$.

Daud MK, Noor RM, Rahman NA, Sidek DS, Mohamad A. (2010) The effect of mild hearing loss on academic performance in primary school children. Int J Pediatr Otorhinolaryngol 74:67-70.

Davis A, Smith P, Ferguson M, Stephens D, Gianopoulos I. (2007) Acceptability, benefit and costs of early screening for hearing disability: a study of potential screening tests and models. Health Technol Assess 11(42):1-294.

Duthey B. (2013) Background paper 6.21 hearing loss [PDF document]. Priority Medicines for Europe and the World "A Public Health Approach to Innovation": Update on 2004 Background Paper from http://www.who.int/medicines/areas/priority_medicines/ BP6_21Hearing.pdf?ua=1. Accessed January 25, 2016.

Harrison JA, Mullen PD, Green LW. (1992) A meta-analysis of studies of the health belief model with adults. Health Educ Res $7: 107-116$

Hearing Loss Association of America (HLAA). (2015) The HLAA Facts \& Statistics. Bethesda, MD. http://www.hearingloss.org/ sites/default/files/docs/HearingLoss_Facts_Statistics.pdf. Accessed January 14, 2016.

Hendershot C, Pakulski LA, Thompson A, Dowling J, Price JH. (2011) School nurses' role in identifying and referring children at risk of noise-induced hearing loss. J Sch Nurs 27(5):380-390.

Hogan A, Shipley M, Strazdins L, Purcell A, Baker E. (2011) Communication and behavioural disorders among children with hearing loss increases risk of mental health disorders. Aust $N Z \mathrm{~J}$ Public Health 35(4):377-383.

Humes LE, Rogers SE, Quigley TM, Main AK, Kinney DL, Herring C. (2017) The effects of service-delivery model and purchase price on hearing-aid outcomes in older adults: a randomized double-blind placebo-controlled clinical trial. Am J Audiol 26(1): 53-79.

Janz NK, Becker MH. (1984) The health belief model: a decade later. Health Educ Q 11(1):1-47.

Kochkin S. (2005) MarkeTrak VII: Hearing Loss Population Tops 31 Million, Hearing Review, 12. http://www.hearingreview.com/ articles.ASP?ArticleId=H0507F01. Accessed February 15, 2016.

Kochkin S. (2013) The Impact of Treated Hearing Loss on Quality of Life. Better Hearing Institute: Aural Education and Counseling. http://www.betterhearing.org/sites/default/files/quality_of_life.pdf. Accessed January 7, 2016.

Kochkin S, Rogin CM. (2000) Quantifying the Obvious: The Impact of Hearing Instruments on Quality of Life, Hearing Review. http:// www.betterhearing.org/sites/default/files/hearingpedia/Hearing_ aids_and_quality_of_life_NCOA.pdf. Accessed January 7, 2016.

Larson VD, Williams DW, Henderson WG, Luethke LE, Beck LB, Noffsinger D, Wilson RH, Dobie RA, Haskell GB, Bratt GW, Shanks JE, Stelmachowicz P, Studebaker GA, Boysen AE, Donahue A, Canalis R, Fausti SA, Rappaport BZ. (2000) Efficacy of three commonly used hearing aid circuits: a crossover trial. $J$ Am Med Assoc 284:1806-1813. 
Lin FR, Metter EJ, O'Brien RJ, Resnick SM, Zonderman AB, Ferrucci L. (2011) Hearing loss and incident dementia. Arch Neurol 68(2):214-220.

National Academies of Sciences, Engineering, and Medicine (NASEM). (2016) Hearing Health Care for Adults: Priorities for Improving Access and Affordability. Washington, DC: The National Academies Press.

National Institute on Deafness and Other Communication Disorders (NIDOCD). (2015) Noise-Induced Hearing Loss. Bethesda, MD: U.S. Department of Health and Human Services.

National Institutes of Health (NIH). (2010) Hearing Aids: Fact Sheet. https://report.nih.gov/nihfactsheets/Pdfs/HearingAids(NIDCD). pdf. Accessed January 25, 2016.
Pittman A, Vincent K, Carter L. (2009) Immediate and long-term effects of hearing loss on the speech perception of children. $J$ Acoust Soc Am 126(3):1477-1485.

Prochaska JO, DiClemente CC, Norcross J. (1992) In search of how people change: applications to addictive behaviors. Am Psychol 47: 1102-1114.

Schoenborn CA, Heyman K. (2008) Health Disparities among Adults with Hearing Loss: United States, 2000-2006. NCHS Health E-Stats. http://www.cdc.gov/nchs/data/hestat/hearing00-06/ hearing00-06.pdf

Shargorodsky J, Curhan SG, Curhan GC, Eavey R. (2010) A prospective study of cardiovascular risk factors and incident hearing loss in men. Laryngoscope 120(9):1887-1891. 